\title{
REGULAR RINGS AND INTEGRAL EXTENSION OF A REGULAR RING
}

\author{
EDWARD T. WONG
}

\begin{abstract}
In this paper we show that a ring (not necessarily commutative) with identity element and without nonzero nilpotent elements is a von Neumann regular ring if every completely prime ideal is a maximal right ideal. Using this result, we show an integral extension (not necessarily commutative) without nonzero nilpotent elements of a regular ring is itself a regular ring.
\end{abstract}

Let $R$ be a ring with unit 1. A proper ideal $P$ in $R$ is called a completely prime ideal if $a b \in P$ implies either $a \in P$ or $b \in P$. A proper ideal $Q$ in $R$ is called a semicompletely prime ideal if $a^{n} \in Q$, for some integer $n$, then $a \in Q$. Andrunakievič and Rjabuhin [1] proved that if $Q$ is a semicompletely prime ideal and $M^{*}$ is a maximal $m$-system where $M^{*} \cap Q=$ $\varnothing$, then the complement of $M^{*}$ in $R$ is a completely prime ideal.

For any $x \neq 0$ in $R$, let $J(x)$ be the right annihilator ideal of $x$ in $R$ and $A(x R)=\{y \in R \mid$ there exists $r \in R, x r \neq 0$ and $(x r) y=0\} . A(x R)$ is the set of zero-divisors on the right $R$-module $x R . J(x) \subset A(x R)$ and the complement of $A(x R)$ in $R$ is a multiplicatively closed system.

LEMMA 1. If $R$ has no nonzero nilpotent elements then $J(x)$ is a semicompletely prime ideal and $a b \in J(x)$ implies $b a \in J(x)$.

Proof. In a ring without nonzero nilpotent elements, $y z=0$ implies $z y=0$. Since $(z y)^{2}=z(y z) y$. Therefore $J(x)$ is also the left annihilator ideal of $x$ and hence an ideal in $R$. If $b^{n} \in J(x)$, for some integer $n>1$, then $0=x b^{n}=x b^{n-1} b=b x b^{n-1}$. If $n=2$ then $b x b x=0$. This implies $b x=0$ and $b \in J(x)$. If $n>2$ then $0=b x b^{n-2} b$ and hence $\left(b x b^{n-2}\right)^{2}=0$. Thus $b x b^{n-2}=$ $x b^{n-1}=0$. Continuing this process, after a finite number of steps, we obtain $x b=0 . J(x)$ is a semicompletely prime ideal. If $a b \in J(x)$ then $(b a)^{2}=b(a b) a \in J(x)$ and hence $b a \in J(x)$.

THEOREM 1. Let $R$ be a ring with no nonzero nilpotent elements. For any $x \neq 0$ in $R$, if $P$ is a minimal completely prime ideal containing $J(x)$ then $P \subset A(x R)$.

Received by the editors March 25, 1971.

AMS 1969 subject classifications. Primary 1650, 1680.

Key words and phrases. Completely prime ideal, semicompletely prime ideal, $m$ system, multiplicatively closed system, regular ring, integral extension.

(C) American Mathematical Society 1972 
Proof. Let $M=\left\{\left(a_{1} m_{1}\right) \cdots\left(a_{n} m_{n}\right) \mid n\right.$ a natural number, $a_{i} \notin P$, $\left.m_{i} \notin A(x R)\right\} . M$ is a multiplicatively closed system and contains the complement of $P$ and the complement of $A(x R) . M \cap J(x)=\varnothing$. Since if $\left(a_{1} m_{1}\right) \cdots\left(a_{n-1} m_{n-1}\right)\left(a_{n} m_{n}\right) \in(M \cap J(x))$ and $n>1$ then

$$
\left(a_{1} m_{1}\right) \cdots\left(a_{n-1} m_{n-1}\right) a_{n} \in J(x) .
$$

By Lemma 1, $\left(\left(a_{n} a_{1}\right) m_{1}\right) \cdots\left(a_{n-1} m_{n-1}\right) \in J(x) .\left(a_{n} a_{1}\right) \notin P$ because $P$ is completely prime. Continuing this process, eventually we will obtain an $a m_{1} \in(J(x) \cap M)$ where $a \notin P$. But this is impossible, since $(x a) m_{1}=0$ implies $x a=0 . a \in J(x) \subset P$. Let $M^{*}$ be a maximal $m$-system where $M \subset M^{*}$ and $M^{*} \cap J(x)=\varnothing$. By [1], $C\left(M^{*}\right)=P^{*}$, the complement of $M^{*}$, is a completely prime ideal. $J(x) \subset P^{*} \subset P$ and $P^{*} \subset A(x R)$. By the minimality of $P, P=P^{*} \subset A(x R)$.

LEMMA 2. Let $R$ be a ring with unit 1 and no nonzero nilpotent elements. If every completely prime ideal of $R$ is a maximal right ideal then every nonunit element of $R$ is contained in a completely prime ideal.

Proof. If $x$ is not a unit in $R$ then $x R \neq R$, because a right inverse of an element is the inverse of the element in a ring with no nonzero nilpotent elements. Let $M=\{x r+1 \mid r \in R\} . M$ is a multiplicatively closed system and $0 \notin M$. Since the zero ideal is semicompletely prime, there exists a completely prime ideal $P$ where $P \cap M=\varnothing$. If $x R \leftarrow P$, then $x R+P=R . x r+p=$ 1 for some $r \in R$ and some $p \in P$. $p=x(-r)+1$ is in $M$, a contradiction.

THEOREM 2. If $R$ has no nonzero nilpotent elements and every completely prime ideal is a maximal right ideal, then any nonzero-divisor is a unit.

Proof. If $x \in R$ with $J(x)=0$, then by Theorem 1 , any completely prime ideal consists of zero-divisors. By the above lemma, $x$ must be a unit.

A ring is called a regular ring (von Neumann), if for each $x$ there exists $y$ such that $x y x=x$. In a regular ring, it is easy to show every completely prime ideal is a maximal right ideal. A commutative ring is a regular ring if and only if it has no nonzero nilpotent elements and every prime ideal is a maximal ideal [2, p. 63].

THEOREM 3. Let $R$ be a ring with unit 1 and no nonzero nilpotent elements. If every completely prime ideal of $R$ is a maximal right ideal then $R$ is a regular ring. Furthermore, for each $x \in R$ there exists a unit $u$ such tilat $x^{2} u=x$.

Proof. $\quad x \neq 0$ in $R, R / J(x)$ is again a ring without nonzero nilpotent elements and every completely prime ideal is a maximal right ideal. 
$\bar{x}=x+J(x)$ is not a zero-divisor in $R / J(x)$. Since $x b \in J(x)$ implies $b x \in$ $J(x),(x b)^{2}=x b x b=0$ implies $b \in J(x)$. By Theorem 2, there exists $r \in R$ and $p \in J(x), x r+p=1$. $x r x=x^{2} r=x$. $R$ is a regular ring.

In a ring with no nonzero nilpotent elements, every idempotent is central. Let $e=x r$ where $x^{2} r=x$ and $u=(1-e)+e r$. It is easy to verify $J(u)=0$ and $x^{2} u=x$. By Theorem 2, $u$ is a unit.

COROllaRY 1. Let $R$ be a ring with unit 1 and no nonzero nilpotent elements. $R$ is a regular ring if $R / P$ is regular for every prime ideal $P$ in $R .^{1}$

Proof. Let $Q$ be a completely prime ideal in $R$. $Q$ is prime. $R / Q$ is a division ring, because $R / Q$ is von Neumann regular and has no nonzerodivisor. Therefore $Q$ is a maximal right (and left) ideal in $R$ and $R$ is a regular ring.

COROLlARY 2. A commutative ring with a unit is a regular ring if and only if it has no nonzero nilpotent elements and every prime ideal is a maximal ideal.

Integral extension. $S$ is a ring with identity element and $R$ is a subring of $S$ containing the identity element of $S$. We call $S$ an integral extension of $R$, or $S$ is integral over $R$, if for each $s \in S$, there exist a positive integer $n$ and elements $a_{n-1}, \cdots, a_{0}$ in $R$ such that $s^{n}+a_{n-1} s^{n-1}+\cdots+a_{0}=0$.

THEOREM 4. Let $S$ be an integral extension of $R$. If $R$ is a regular ring then any completely prime ideal $Q$ in $S$ is a maximal right ideal in $S$.

Proof. Let $P=Q \cap R$. $P$ is a completely prime ideal in $R$. Since $R$ is a regular ring, $R / P$ is a division ring. $S / Q$ is an integral domain (not necessarily commutative) and is an integral extension of $R / P$. $S / Q$ must be a division ring and hence $Q$ is a maximal right ideal in $S$.

Together with Theorem 3, we have:

THEOREM 5. An integral extension with no nonzero nilpotent elements of a regular ring is itself a regular ring.

\section{REFERENCES}

1. V. A. Andrunakievič and Ju. M. Rjabuhin, Rings without nilpotent elements and completely simple ideals, Dokl. Akad. Nauk SSSR 180 (1968), 9-11=Soviet Math. Dokl. 9 (1968), 565-567. MR 37 \#6320.

2. I. Kaplansky, Commutative rings, Allyn and Bacon, Boston, Mass., 1970. MR 40 \#7234.

Department of Mathematics, Oberlin College, Oberlin, Ohio 44704

1 This result was discovered by I. N. Herstein. Herstein proves it from a different approach and without assuming the existence of an identity element. 